TRANSACTIONS OF THE

AMERICAN MATHEMATICAL SOCIETY

Volume 352, Number 6, Pages 2765-2787

S 0002-9947(99)02544-1

Article electronically published on December 10, 1999

\title{
INVERSE SPECTRAL ANALYSIS WITH PARTIAL INFORMATION ON THE POTENTIAL, II. THE CASE OF DISCRETE SPECTRUM
}

\author{
FRITZ GESZTESY AND BARRY SIMON
}

\begin{abstract}
We discuss results where the discrete spectrum (or partial information on the discrete spectrum) and partial information on the potential $q$ of a one-dimensional Schrödinger operator $H=-\frac{d^{2}}{d x^{2}}+q$ determine the potential completely. Included are theorems for finite intervals and for the whole line. In particular, we pose and solve a new type of inverse spectral problem involving fractions of the eigenvalues of $H$ on a finite interval and knowledge of $q$ over a corresponding fraction of the interval. The methods employed rest on Weyl $m$-function techniques and densities of zeros of a class of entire functions.
\end{abstract}

\section{INTRODUCTION}

In 1978, Hochstadt and Lieberman [16] proved the following remarkable theorem:

Theorem 1.1. Let $h_{0} \in \mathbb{R}, h_{1} \in \mathbb{R} \cup\{\infty\}$, and assume $q_{1}, q_{2} \in L^{1}((0,1))$ to be real-valued. Consider the Schrödinger operators $H_{1}, H_{2}$ in $L^{2}((0,1))$ given by

$$
H_{j}=-\frac{d^{2}}{d x^{2}}+q_{j}, \quad j=1,2,
$$

with the boundary conditions

$$
\begin{aligned}
& u^{\prime}(0)+h_{0} u(0)=0, \\
& u^{\prime}(1)+h_{1} u(1)=0 .
\end{aligned}
$$

Let $\sigma\left(H_{j}\right)=\left\{\lambda_{j, n}\right\}$ be the (necessarily simple) spectra of $H_{j}, j=1,2$. Suppose that $q_{1}=q_{2}$ (a.e.) on $\left[0, \frac{1}{2}\right]$ and that $\lambda_{1, n}=\lambda_{2, n}$ for all $n$. Then $q_{1}=q_{2}$ (a.e.) on $[0,1]$.

Here, in obvious notation, $h_{1}=\infty$ in (1.1b) singles out the Dirichlet boundary condition $u(1)=0$.

For each $\varepsilon>0$, there are simple examples where $q_{1}=q_{2}$ on $\left[0, \frac{1}{2}-\varepsilon\right]$ and $\sigma\left(H_{1}\right)$ $=\sigma\left(H_{2}\right)$ but $q_{1} \neq q_{2}$. (Choose $h_{0}=-h_{1}, q_{1}(x)=0$ for $x \in\left(0, \frac{1}{2}-\varepsilon\right] \cup\left[\frac{1}{2}, 1\right]$ and nonzero on $\left(\frac{1}{2}-\varepsilon, \frac{1}{2}\right)$, and $q_{2}(x)=q_{1}(1-x)$. See also Theorem $\mathrm{I}^{\prime}$ in the appendix of [37].)

Later refinements of Theorem 1.1 in [13] and [37] (see also the summary in [35]) showed that the boundary condition for $H_{1}$ and $H_{2}$ at $x=1$ need not be assumed a priori to be the same, and that if $q$ is continuous, then one only needs $\lambda_{1, n}=\lambda_{2, m(n)}$

Received by the editors October 9, 1997.

2000 Mathematics Subject Classification. Primary 34A55, 34L40; Secondary 34 B20.

This material is based upon work supported by the National Science Foundation under Grant Nos. DMS-9623121 and DMS-9401491. 
for all values of $n$ but one. (37] claims the result does not require continuity of $q$, but we will see in Section 3 that this assertion is false.) The same boundary condition for $H_{1}$ and $H_{2}$ at $x=0$, however, is crucial for Theorem 1.1 to hold (see [13], [33]).

Moreover, analogs of Theorem 1.1 for certain Schrödinger operators are considered in [19, and the interval $\left[0, \frac{1}{2}\right]$ replaced by different subsets of $[0,1]$ was studied in [18] (see also [31, Ch. 4). Reconstruction techniques for $q(x)$ in this context are discussed in 34.

Our purpose in this paper is to provide a new approach to Theorem 1.1 that we feel is more transparent and, moreover, capable of vast generalizations. To state our generalizations, we will introduce a shorthand notation to paraphrase Theorem 1.1 by saying " $q$ on $\left[0, \frac{1}{2}\right]$ and the eigenvalues of $H$ uniquely determine $q$." This is just a shorthand notation for saying $q_{1}=q_{2}$ if the obvious conditions hold.

Unless explicitly stated otherwise, all potentials $q, q_{1}$, and $q_{2}$ will be real-valued and in $L^{1}((0,1))$ for the remainder of this paper. Moreover, to avoid too many case distinctions in the proofs below, we shall assume $h_{0}, h_{1} \in \mathbb{R}$ in (1.1) throughout the main body of this paper. In particular, for $h_{0}, h_{1} \in \mathbb{R}$ we index the corresponding eigenvalues $\lambda_{n}$ of $H$ by $n \in \mathbb{N}_{0}=\mathbb{N} \cup\{0\}$. The case of Dirichlet boundary conditions, where $h_{0}=\infty$ and/or $h_{1}=\infty$, will be dealt with in Appendix A.

Here are some of the generalizations we will prove for Schrödinger operators on $[0,1]$ :

Theorem 1.2. Let $H=-\frac{d^{2}}{d x^{2}}+q$ in $L^{2}((0,1))$ with boundary conditions (1.1) and $h_{0}, h_{1} \in \mathbb{R}$. Suppose $q$ is $C^{2 k}\left(\left(\frac{1}{2}-\varepsilon, \frac{1}{2}+\varepsilon\right)\right)$ for some $k=0,1, \ldots$ and for some $\varepsilon>0$. Then $q$ on $\left[0, \frac{1}{2}\right], h_{0}$, and all the eigenvalues of $H$ except for $(k+1)$ uniquely determine $h_{1}$ and $q$ on all of $[0,1]$.

Remarks. 1. The case $k=0$ in Theorem 1.2 is due to Hald [13].

2. In the non-shorthand form of this theorem, we mean that both $q_{1}$ and $q_{2}$ are $C^{2 k}$ near $x=\frac{1}{2}$.

3. One need not know which eigenvalues are missing. Since the eigenvalues asymptotically satisfy

$$
\lambda_{n}=(\pi n)^{2}+2\left(h_{1}-h_{0}\right)+\int_{0}^{1} d x q(x)+o(1) \quad \text { as } n \rightarrow \infty,
$$

given a set of candidates for the spectrum, one can tell how many are missing.

4. For the sake of completeness we mention the precise definition of $H$ in $L^{2}((0,1))$ for real-valued $q \in L^{1}((0,1))$ and boundary condition parameters $h_{0}, h_{1} \in$ $\mathbb{R} \cup\{\infty\}$ in (1.1):

$$
\begin{gathered}
H=-\frac{d^{2}}{d x^{2}}+q \\
D(H)=\left\{g \in L^{2}((0,1)) \mid g, g^{\prime} \in A C([0,1]) ;\left(-g^{\prime \prime}+q g\right) \in L^{2}((0,1)) ;\right. \\
\left.\quad g^{\prime}(0)+h_{0} g(0)=0, g^{\prime}(1)+h_{1} g(1)=0\right\},
\end{gathered}
$$

where $A C([0,1])$ denotes the set of absolutely continuous functions on $[0,1]$ and $h_{x_{0}}=\infty$ represents the Dirichlet boundary condition $g\left(x_{0}\right)=0$ for $x_{0} \in\{0,1\}$ in $(1.3)$. 
In Section 3, we discuss examples which show that Theorem 1.2 is optimal in the sense that if $q$ is only assumed to be $C^{2 k-1}$ near $x=\frac{1}{2}$ for some $k \geq 1$, then it is not uniquely determined by $q \uparrow\left[0, \frac{1}{2}\right]$ and all the eigenvalues but $(k+1)$.

Theorem 1.2 works because the condition that $q$ is $C^{2 k}$ near $x=\frac{1}{2}$ gives us partial information about $q$ on $\left[\frac{1}{2}, 1\right]$; namely, we know $q\left(\frac{1}{2}\right), q^{\prime}\left(\frac{1}{2}\right), \ldots, q^{(2 k)}\left(\frac{1}{2}\right)$ computed on $\left[\frac{1}{2}, 1\right]$ since we can compute them on $\left[0, \frac{1}{2}\right]$. This suggests that knowing $q$ on more than $\left[0, \frac{1}{2}\right]$ should let one dispense with a finite density of eigenvalues. That this is indeed the case is the content of the following theorem:

Theorem 1.3. Let $H=-\frac{d^{2}}{d x^{2}}+q$ in $L^{2}((0,1))$ with boundary conditions (1.1) and $h_{0}, h_{1} \in \mathbb{R}$. Then $q$ on $\left[0, \frac{1}{2}+\frac{\alpha}{2}\right]$ for some $\alpha \in(0,1), h_{0}$, and a subset $S \subseteq \sigma(H)$ of all the eigenvalues $\sigma(H)$ of $H$ satisfying

$$
\#\left\{\lambda \in S \mid \lambda \leq \lambda_{0}\right\} \geq(1-\alpha) \#\left\{\lambda \in \sigma(H) \mid \lambda \leq \lambda_{0}\right\}+\frac{\alpha}{2}
$$

for all sufficiently large $\lambda_{0} \in \mathbb{R}$, uniquely determine $h_{1}$ and $q$ on all of $[0,1]$.

Remarks. 1. As a typical example, knowing slightly more than half the eigenvalues and knowing $q$ on $\left[0, \frac{3}{4}\right]$ determines $q$ uniquely on all of $[0,1]$. To the best of our knowledge, Theorem 1.3 solves a new type of inverse spectral problem. In particular, we are not aware of any inverse spectral result involving fractions of the set of eigenvalues as in (1.4).

2. As in the case $\alpha=0$, we have an extension of the same type as Theorem 1.2. Explicitly, if $q$ is assumed to be $C^{2 k}$ near $x=\frac{1}{2}+\frac{\alpha}{2}$, we only need

$$
\#\left\{\lambda \in S \mid \lambda \leq \lambda_{0}\right\} \geq(1-\alpha) \#\left\{\lambda \in \sigma(H) \mid \lambda \leq \lambda_{0}\right\}+\frac{\alpha}{2}-(k+1)
$$

instead of (1.4).

We can also derive results about problems on all of $\mathbb{R}$. In Section 5 , we will prove

Theorem 1.4. Suppose that $q \in L_{\mathrm{loc}}^{1}(\mathbb{R})$ obeys

(i) $q(x) \geq C|x|^{2+\varepsilon}-D$ for some $C, \varepsilon, D>0$, and

(ii) $q(-x) \geq q(x) \quad x \geq 0$.

Then $q$ on $[0, \infty)$ and the spectrum of $H=-\frac{d^{2}}{d x^{2}}+q$ in $L^{2}(\mathbb{R})$ uniquely determine $q$ on all of $\mathbb{R}$.

In Section 5, we will also present further conjectures and explain how condition (i) is related to the class of entire functions of order less than one.

All these results are related to two other papers we have written. In [12, we consider, among other topics, analogs of Theorems 1.1 and 1.3 for finite tridiagonal (Jacobi) matrices extending a result in 15]. The approach there is very similar to the current one, except that the somewhat subtle theorems on zeros of entire functions in this paper are replaced by the elementary fact that a polynomial of degree at most $N$ with $N+1$ zeros must be identically zero. In [11], we consider results related to Theorem 1.4 in that, for Schrödinger operators on $(-\infty, \infty)$, "spectral" information plus the potential on one of the half-lines determine the potential on all of $(-\infty, \infty)$. In that paper, we consider situations where there are scattering states for some set of energies and the "spectral" data are given by a reflection coefficient on a set of positive Lebesgue measure in the a.c. spectrum of $H$. The approach is not as close to this paper as is [12], but $m$-function techniques (see also [10]) are critical in all three papers. 
Hochstadt and Lieberman 16] use the details of inverse spectral theory in their proof. In a sense, we only use the main uniqueness theorem of that theory due to Marchenko 28], which we now describe. For $q \in L^{1}((a, b))$ real-valued, $-\infty<a<$ $b<\infty$, consider

$$
-u^{\prime \prime}+q u=z u
$$

with the boundary condition

$$
u^{\prime}(b)+h_{b} u(b)=0
$$

at $x=b$. Let $u_{+}(z, x)$ denote the solution of this equation, normalized, say, by $u_{+}(z, b)=1$. The $m_{+}$-function is then defined by

$$
m_{+}(z, a)=\frac{u_{+}^{\prime}(z, a)}{u_{+}(z, a)}
$$

Similarly, given a boundary condition at $x=a$,

$$
u^{\prime}(a)+h_{a} u(a)=0,
$$

we define the solution $u_{-}(z, x)$ of $(1.6)$ normalized by $u_{-}(z, a)=1$ and then define

$$
m_{-}(z, b)=-\frac{u_{-}^{\prime}(z, b)}{u_{-}(z, b)} .
$$

The differing signs in (1.8) and (1.10) are picked so that both $m_{+}$and $m_{-}$are Herglotz functions, that is, $m_{ \pm}: \mathbb{C}_{+} \rightarrow \mathbb{C}_{+}$are analytic (in our present context where $-\infty<a<b<\infty, m_{ \pm}$are even meromorphic on $\left.\mathbb{C}\right), \mathbb{C}_{+}$being the open complex upper half-plane. In particular,

$$
\operatorname{Im}(z)>0 \Longrightarrow \operatorname{Im}\left(m_{-}(z, b)\right)>0, \quad \operatorname{Im}\left(m_{+}(z, a)\right)>0 .
$$

Marchenko's [28] fundamental uniqueness theorem of inverse spectral theory then reads as follows:

Theorem 1.5. $m_{+}(z, a)$ uniquely determines $h_{b}$ as well as $q$ (a.e.) on $[a, b]$.

If $q \in L_{\mathrm{loc}}^{1}([a, \infty))$ is real-valued (with $|a|<\infty$ ) and $-\frac{d^{2}}{d x^{2}}+q$ is in the limit point case at infinity, one can still define a unique $m_{+}(z, a)$ function, but now for $\operatorname{Im}(z) \neq 0$ rather than all $z \in \mathbb{C}$. For $\operatorname{such} z$, there is a unique function $u_{+}(z, \cdot)$ which is $L^{2}$ at infinity (unique up to an overall scale factor which drops out of $m_{+}(z, a)$ defined by (1.8)). Again, one has the following uniqueness result, independently proved by Borg [3] and Marchenko [28]

Theorem 1.6. $m_{+}(z, a)$ uniquely determines $q($ a.e. $)$ on $[a, \infty)$.

It is useful to have $m_{-}(z, b)$ because of the following basic fact:

Theorem 1.7. Let $H=-\frac{d^{2}}{d x^{2}}+q$ be a Schrödinger operator in $L^{2}((a, b))$ with boundary conditions (1.7), (1.9), and let $G(z, x, y)$ be the integral kernel of $(H-z)^{-1}$. Suppose $c \in(a, b)$, and let $m_{+}(z, c)$ be the corresponding $m_{+}$-function for $[c, b]$ and $m_{-}(z, c)$ the $m_{-}$-function for $[a, c]$. Then

$$
G(z, c, c)=-\frac{1}{m_{+}(z, c)+m_{-}(z, c)} .
$$


Theorems 1.5 and 1.6 are deep facts; Theorem 1.7 is an elementary calculation from the explicit formula for the integral kernel of $(H-z)^{-1}$,

$$
G(z, x, y)=\frac{u_{-}(z, \min (x, y)) u_{+}(z, \max (x, y))}{W\left(u_{-}(z), u_{+}(z)\right)(x)}
$$

where $W(\cdot, \cdot)$ is the Wronskian defined by

$$
W(f, g)(x)=f^{\prime}(x) g(x)-f(x) g^{\prime}(x) .
$$

An analog of Theorem 1.7 holds in case $[a, b]$ is replaced by $(-\infty, \infty)$.

We can now describe the strategy of our proofs of Theorems 1.1-1.4. G(z,c,c) has poles at the eigenvalues of $H$ (this is not quite true; see below), so by (1.12), at eigenvalues $\lambda_{n}$ of $H$ :

$$
m_{+}\left(\lambda_{n}, c\right)=-m_{-}\left(\lambda_{n}, c\right) .
$$

If we know $q$ on a left partial interval $[a, c]$ and we know some eigenvalue $\lambda_{n}$, then we know $m_{-}(z, c)$ exactly; so by (1.13), we know the value of $m_{+}\left(\lambda_{n}, c\right)$ at the point $\lambda_{n}$. In Appendix B we discuss when knowing the values of $f\left(\lambda_{n}\right)$ of an analytic function of the type of the $m$-functions uniquely determines $f(z)$. If $m_{+}(z, c)$ is determined, then by Theorem 1.5, $q$ is determined on $[a, b]$, and so is $h_{b}$.

So the logic of the argument for a theorem like Theorem 1.1 is the following:

(i) $q$ on $\left[0, \frac{1}{2}\right]$ and $h_{0}$ determine $m_{-}\left(z, \frac{1}{2}\right)$ by direct spectral theory.

(ii) The $\lambda_{n}$ and (1.13) determine $m_{+}\left(\lambda_{n}, \frac{1}{2}\right)$, and then, by suitable theorems in complex analysis, $m_{+}\left(z, \frac{1}{2}\right)$ is uniquely determined for all $z$.

(iii) $m_{+}\left(z, \frac{1}{2}\right)$ uniquely determines $q$ (a.e.) on $\left[\frac{1}{2}, 1\right]$ and $h_{1}$ by inverse spectral theory.

It is clear from this approach why $h_{0}$ is required and $h_{1}$ is free in the context of Theorem 1.1 (see [33] for examples where $h_{1}$ and $q \uparrow\left[0, \frac{1}{2}\right]$ do not determine $q$ ); without $h_{0}$ we cannot compute $m_{-}\left(z, \frac{1}{2}\right)$ and so start the process.

As indicated before (1.13), $G(z, c, c)$ may not have a pole at an eigenvalue $\lambda_{n}$ of $H$. It will if $u_{n}(c) \neq 0$, but if $u_{n}(c)=0$, then $G(z, c, c)=0$ rather than $\infty$. Here $u_{n}$ denotes the eigenfunction of $H$ associated with the (necessarily simple) eigenvalue $\lambda_{n}$. Nevertheless, (1.13) holds at points where $u_{n}(c)=0$, since then $u_{-}(c)=u_{+}(c)=0$, and so both sides of (1.13) are infinite. (In spite of (1.13), $m_{+}+m_{-}$is also infinite at $z=\lambda_{n}$ and so $G\left(\lambda_{n}, c, c\right)=0$.) We summarize this discussion in the following:

Theorem 1.8. For any $c \in(a, b)$, (1.13) holds at any eigenvalue $\lambda_{n}$ of $H_{[a, b]}$ (with the possibility of both sides of (1.13) being infinite).

An alternative way of proving (1.13) is that $\lambda_{n}$ is an eigenvalue if and only if the Wronskian of $u_{+}$and $u_{-}$is zero, which is precisely (1.13).

Here is a sketch of the contents of this paper. In Section 2 we present our proofs of Theorems 1.1 and 1.2. In Section 3 we discuss an example that delimits Theorem 1.2 and shows that Theorem 1.2 is optimal with respect to smoothness conditions on $q$. In Section 4 we prove Theorem 1.3, and in Section 5 we prove Theorem 1.4. Appendix A is devoted to the case of Dirichlet boundary conditions, and Appendix B presents some facts on entire functions that are necessary to prove our principal results.

Refinements of the results of this paper can be found in [6], [7]. 


\section{Theorems For a Half Interval}

In this section, we will prove the original Hochstadt-Lieberman theorem (Theorem 1.1) and our extension of it (Theorem 1.2) for $h_{0}, h_{1} \in \mathbb{R}$. Consider a problem on $[0,1]$ with boundary condition $(1.1 \mathrm{~b})$ at $x=1$. Let $u_{+}(z, x)$ be defined by $-u_{+}^{\prime \prime}+q u_{+}=z u_{+}$and

$$
u_{+}^{\prime}(z, 1)=-h_{1}, \quad u_{+}(z, 1)=1 .
$$

Then $u_{+}$is known to have the following properties:

(1) For each $x \in[0,1], u_{+}(z, x), u_{+}^{\prime}(z, x)$ are entire functions of $z$. (This follows from the fact that $u_{+}(z, 1)=1$ and $u_{+}^{\prime}(z, 1)=-h_{1}$ are independent of $z$; see, e.g., 4], Theorem I.8.4, Problem I.7, and p. 226.)

$$
\begin{aligned}
& u_{+}(z, x)=\cos (\sqrt{z}(1-x))+O\left(\frac{e^{\operatorname{Im}(\sqrt{z})(1-x)}}{\sqrt{z}}\right), \\
& u_{+}^{\prime}(z, x)=\sqrt{z} \sin (\sqrt{z}(1-x))+O\left(e^{\operatorname{Im}(\sqrt{z})(1-x)}\right)
\end{aligned}
$$

as $|z| \rightarrow \infty$ for all $x \in[0,1]$, where $\sqrt{z}$ is the square root branch with $\operatorname{Im}(\sqrt{z}) \geq 0$ (see, e.g., [29], Sect. 1.4).

(3) The zeros of $u_{+}(\cdot, x)$ and $u_{+}^{\prime}(\cdot, x)$ are all real for any $x \in[0,1]$ and they all lie in some $\lambda$-interval $[c, \infty)$ (this is because these zeros are eigenvalues of self-adjoint boundary value problems for Schrödinger operators in $L^{2}((0,1))$ bounded from below).

The final pair of preliminary results we need concerns the high-energy asymptotics of the $m_{+}$-function,

$$
m_{+}(z, x)=\frac{u_{+}^{\prime}(z, x)}{u_{+}(z, x)} .
$$

(4) It is known [1], 8] that under the general hypothesis $q \in L^{1}((0,1))$,

$$
m_{+}(z, x)^{-1}=-i(\sqrt{z})^{-1}\left(1+o\left(z^{-1 / 2}\right)\right)
$$

uniformly in $x \in[0,1-\delta], \delta>0$ as $|z| \rightarrow \infty$ in any sector $\varepsilon<\operatorname{Arg}(z)<$ $\pi-\varepsilon, \varepsilon>0$.

(5) If $q$ is $C^{2 k}$ near $x_{0} \in(0,1), k=0,1,2, \ldots$, then $m_{+}\left(z, x_{0}\right)$ and $m_{+}\left(z, x_{0}\right)^{-1}$ are known to have asymptotic expansions of the form (see [5])

$$
\begin{aligned}
m_{+}\left(z, x_{0}\right)=i(\sqrt{z})\left(\sum_{\ell=0}^{2 k+2} C_{\ell}\left(x_{0}\right) z^{-\ell / 2}+o\left(z^{-k-1}\right)\right), & C_{0}\left(x_{0}\right)=1, \\
m_{+}\left(z, x_{0}\right)^{-1}=-i(\sqrt{z})^{-1}\left(\sum_{\ell=0}^{2 k+2} D_{\ell}\left(x_{0}\right) z^{-\ell / 2}+o\left(z^{-k-1}\right)\right), & D_{0}\left(x_{0}\right)=1,
\end{aligned}
$$

as $|z| \rightarrow \infty$ in any sector $\varepsilon<\operatorname{Arg}(z)<\pi-\varepsilon, \varepsilon>0$, where $C_{\ell}\left(x_{0}\right)$ and $D_{\ell}\left(x_{0}\right)$ are universal functions of $q\left(x_{0}\right), \ldots, q^{(\ell-2)}\left(x_{0}\right)$. In fact, $C_{\ell}(x)$ and $D_{\ell}\left(x_{0}\right)$ have a well-known connection to the conserved densities of the KdV hierarchy [9] and they can be computed recursively as follows. Consider the Riccati-type equations for $m_{+}(z, x)$ and $m_{+}(z, x)^{-1}$,

$$
\begin{gathered}
m_{+}^{\prime}(z, x)+m_{+}(z, x)^{2}=q(x)-z \\
{\left[m_{+}(z, x)^{-1}\right]^{\prime}+m_{+}(z, x)^{-2}[q(x)-z]=1 .}
\end{gathered}
$$


Inserting the asymptotic expansions (2.5) and (2.6) into (2.7) and (2.8) then yields the recursion relations

$$
\begin{aligned}
C_{0}(x)= & 1, \quad C_{1}(x)=0, \quad C_{2}(x)=-\frac{1}{2} q(x), \\
C_{j}(x)= & \frac{i}{2} C_{j-1}^{\prime}(x)-\frac{1}{2} \sum_{\ell=1}^{j-1} C_{\ell}(x) C_{j-\ell}(x), \quad j \geq 3, \\
D_{0}(x)= & 1, \quad D_{1}(x)=0, \quad D_{2}(x)=\frac{1}{2} q(x), \\
D_{j}(x)= & \frac{i}{2} D_{j-1}^{\prime}(x)+\frac{1}{2} q(x) \sum_{\ell=0}^{j-2} D_{\ell}(x) D_{j-\ell-2}(x) \\
& -\frac{1}{2} \sum_{\ell=1}^{j-1} D_{\ell}(x) D_{j-\ell}(x), \quad j \geq 3 .
\end{aligned}
$$

With these preliminaries out of the way, let $q$ be given (a.e.) on $\left[0, \frac{1}{2}\right]$ and let $q_{1}, q_{2}$ be two candidates for $q$ extended to all of $[0,1]$. Let $\sigma\left(H_{1}\right)=\left\{\lambda_{1, n}\right\}_{n=0}^{\infty}$ be the set of all the eigenvalues of $H_{1}=-\frac{d^{2}}{d x^{2}}+q_{1}$. Define, for $j=1,2$,

$$
\begin{aligned}
P_{j}(z) & =u_{j,+}\left(z, \frac{1}{2}\right), \\
Q_{j}(z) & =u_{j,+}^{\prime}\left(z, \frac{1}{2}\right), \\
f_{j}(z) & =\frac{P_{j}(z)}{Q_{j}(z)}=m_{j,+}\left(z, \frac{1}{2}\right)^{-1}, \\
g(z) & =u_{1,+}^{\prime}(z, 0)+h_{0} u_{1,+}(z, 0),
\end{aligned}
$$

so that $\left\{\lambda_{1, n}\right\}_{n=0}^{\infty}$ are precisely the zeros of $g(z)$. (Note in this context that $u_{1,+}(z, x)$ satisfies $(1.1 \mathrm{~b})$ at $x=1$ for all $z \in \mathbb{C}$. Thus, if and only if $g(\hat{\lambda})=0$, is it true that $u_{1,+}(\hat{\lambda}, x)$ also satisfies (1.1a) at $x=0$ and hence $\hat{\lambda} \in \sigma\left(H_{1}\right)$.) Here $u_{j, \pm}$ are the corresponding solutions of $-u^{\prime \prime}+q_{j} u=z u$ used in (1.8) and (1.10). By adding a sufficiently large constant to $q_{1}$ and $q_{2}$, we can suppose all the zeros of $P_{j}, Q_{j}$, and $g$ are in $[1, \infty)$.

By (1)-(5) above, we infer:

(a) $P_{j}, Q_{j}$, and $g$ are all of the form (see, e.g., [21], Ch. I; [30], Sect. II.48)

$$
c \prod_{n=0}^{\infty}\left(1-\frac{z}{x_{n}}\right)
$$

for suitable $\left\{x_{n}\right\}_{n=0}^{\infty} \subseteq[1, \infty)$ (which a priori could differ for the five functions).

(b) $P_{j}, Q_{j}$, and $g$ are all bounded by $C_{1} \exp \left(C_{2}|z|^{1 / 2}\right)$ for some $C_{1}, C_{2}>0$.

(c) $\left|f_{1}(i y)-f_{2}(i y)\right|=o\left(|y|^{-1}\right)$ as $y$ (real) $\rightarrow \pm \infty$.

(d) If $q_{j} \in C^{2 k}$ near $x=\frac{1}{2}$, then, as $y$ (real) $\rightarrow \pm \infty$,

$$
\left|f_{1}(i y)-f_{2}(i y)\right|=o\left(|y|^{-(2 k+3) / 2}\right) .
$$

(e) $\left|Q_{j}(i y)\right|=\frac{1}{2}|y|^{1 / 2}\left|\exp \left(\frac{1}{2} \operatorname{Im}(\sqrt{i})|y|^{1 / 2}\right)\right|(1+o(1))$ as $y($ real $) \rightarrow \infty$.

(f) $|g(i y)|=\frac{1}{2}|y|^{1 / 2}\left|\exp \left(\operatorname{Im}(\sqrt{i})|y|^{1 / 2}\right)\right|(1+o(1))$ as $y($ real $) \rightarrow \infty$.

(g) For $n$ sufficiently large, $\inf _{\theta \in[0,2 \pi]}\left|g\left(\left(\pi\left(n+\frac{1}{2}\right)\right)^{2} e^{i \theta}\right)\right| \geq \pi n+O(1)$.

Part (d) holds by (2.6) and (2.10), because $q_{1}^{(\ell)}\left(\frac{1}{2}\right)=q_{2}^{(\ell)}\left(\frac{1}{2}\right)$ for all $0 \leq \ell \leq 2 k$ by the regularity of $q$ near $x=\frac{1}{2}$, and hence the terms $D_{0}\left(x_{0}\right), \ldots, D_{2 k+2}\left(x_{0}\right)$ in $(2.6)$ 
in connection with $q_{1}$ and $q_{2}$ will cancel when inserted into $\left[f_{1}(i y)-f_{2}(i y)\right]$. (g) follows from (2.3) since, by (2.15), the infimum is taken at $\theta=0$.

Proof of Theorem $1.1\left(\right.$ for $\left.h_{0}, h_{1} \in \mathbb{R}\right)$. Define

$$
F(z)=\frac{\left[P_{1}(z) Q_{2}(z)-P_{2}(z) Q_{1}(z)\right]}{g(z)} .
$$

By Theorem 1.8, (1.13) holds at the points $\lambda_{1, n}$. Hence $\frac{Q_{1}(z)}{P_{1}(z)}=\frac{Q_{2}(z)}{P_{2}(z)}$ at $z=\lambda_{1, n}$. Moreover, at points where both sides are infinite, one infers $P_{1}=P_{2}=0$. Thus, the cross ratio $P_{1} Q_{2}-P_{2} Q_{1}$ vanishes at each point where $g$ vanishes, and since $g$ necessarily has simple zeros ( $H_{1}$ has simple spectrum), $F$ is an entire function.

In addition, by (b) and (g), $F(z)$ satisfies

$$
|F(z)| \leq C_{1} \exp \left(C_{2}|z|^{1 / 2}\right),
$$

since (2.17) first holds when $|z|=\left(\pi\left(n+\frac{1}{2}\right)\right)^{2}$ for $n$ sufficiently large (by (f)) and then by the maximum modulus principle for all $z$. By Proposition B.6 (a PhragménLindelöf argument) and (2.17), if we show that $|F(i y)| \rightarrow 0$ as $y \rightarrow \infty$ (y real), then $F \equiv 0$.

But

$$
F(z)=\frac{Q_{1}(z) Q_{2}(z)}{g(z)}\left[f_{1}(z)-f_{2}(z)\right]
$$

so, by (c), (e), and (f), the quantity

$$
|F(i y)|=\frac{1}{2}|y|^{1 / 2}\left[o\left(|y|^{-1}\right)\right](1+o(1))=o\left(|y|^{-1 / 2}\right) \quad \text { as } y(\text { real }) \rightarrow \infty,
$$

goes to zero as required.

Once $F \equiv 0$, we can multiply by $\frac{g(z)}{Q_{1}(z) Q_{2}(z)}$ (which has isolated zeros and poles) to conclude that $f_{1}=f_{2}$, and so, by Theorem $1.5, q_{1}=q_{2}$ (a.e.).

Remark. There is a (patchable) gap in the paper of Hochstadt and Lieberman [16]. They consider an entire function $\psi(z)=H(z) / \omega(z)$ where they show $|H(z)| \leq$ $M \exp \left(\operatorname{Im}(\sqrt{|z|)})\right.$ and $\omega(z)=C \sqrt{z} \sin (\sqrt{z})+O\left(e^{\operatorname{Im}}(\sqrt{z})\right)$ and then claim $|\psi(z)|=$ $O(1 / \sqrt{|z|})$ without comment. Because of the zeros of $\sin (\cdot)$ this is not evident, and one needs a Phragmén-Lindelöf-type argument to complete their proof.

Proof of Theorem 1.2. Let $\left\{\lambda_{\ell}\right\}_{\ell=1}^{k+1}$ be the $k+1$ eigenvalues of $-\frac{d^{2}}{d x^{2}}+q_{1}$ in $L^{2}((0,1))$ which a priori are not assumed to be the same for the two potentials. Now define $\tilde{F}(z)$ by

$$
\tilde{F}(z)=\frac{\left[P_{1}(z) Q_{2}(z)-P_{2}(z) Q_{1}(z)\right]}{g(z)} \prod_{\ell=1}^{k+1}\left(z-\lambda_{\ell}\right)
$$

instead of (2.16).

(2.17) still holds, and as in (2.18) and (2.19), one now infers from (d), (e), and (f) that

$$
|\tilde{F}(i y)|=O\left(|y|^{k+1}\right)|y|^{1 / 2}\left[o\left(|y|^{-(2 k+3) / 2}\right)\right](1+o(1))=o(1) \quad \text { as } y(\text { real }) \rightarrow \infty .
$$

Thus $m_{+}\left(z, \frac{1}{2}\right)$ determines $h_{1}$ (cf. Theorem 1.5), and $q_{1}=q_{2}$ (a.e.) follows as in the previous proof. 


\section{An ExAmple}

Our goal in this section is to construct, for each $k$, a function $q$ on $[0,1]$ with the following properties:

(1) $q$ is $C^{\infty}$ on $\left[0, \frac{1}{2}\right]$ and $\left[\frac{1}{2}, 1\right] ; q$ is $C^{2 k-1}$ on $[0,1]$.

(2) $d^{2 k} q / d x^{2 k}$ is discontinuous at $x=\frac{1}{2}$.

(3) $q=0$ on $\left[0, \frac{1}{2}\right]$.

(4) For a suitable boundary condition parameter $h_{1} \in \mathbb{R}$, the eigenvalues of $-\frac{d^{2}}{d x^{2}}+q$ in $L^{2}((0,1))$ with $u^{\prime}(0)=0, u^{\prime}(1)+h_{1} u(1)=0$ boundary conditions agree with those for $-\frac{d^{2}}{d x^{2}}$ in $L^{2}((0,1))$ with $u^{\prime}(0)=u^{\prime}(1)=0$ boundary conditions with precisely $(k+1)$ exceptions.

For $k=0,(2)$ means that $q$ is discontinuous at $x=\frac{1}{2}$.

This example shows that in Theorem 1.2, one cannot weaken the continuity requirement on $q$. In particular, it provides a counterexample to the claim in Suzuki 37] that Theorem I in his Appendix only requires $q \in L^{1}((0,1))$. Continuity of $q$ at $x=\frac{1}{2}$ is critical for his result to hold.

Our results depend on the following well-known fact:

Proposition 3.1. Suppose that $x_{0}<y_{0}<x_{1}<y_{1}<\cdots$ are given so that for $n$ sufficiently large,

$$
x_{n}=[(2 n) \pi]^{2}, \quad y_{n}=[(2 n+1) \pi]^{2} .
$$

Then, there exist (a unique) $h_{1}$ and a $C^{\infty}$ function $q$ on $\left[\frac{1}{2}, 1\right]$ so that

$$
-\frac{d^{2}}{d x^{2}}+q \quad \text { in } L^{2}\left(\left(\frac{1}{2}, 1\right)\right) ; \quad u^{\prime}\left(\frac{1}{2}\right)=0, u^{\prime}(1)+h_{1} u(1)=0
$$

has eigenvalues $\left\{x_{n}\right\}_{n=0}^{\infty}$ and

$$
-\frac{d^{2}}{d x^{2}}+q \quad \text { in } L^{2}\left(\left(\frac{1}{2}, 1\right)\right) ; \quad u\left(\frac{1}{2}\right)=0, u^{\prime}(1)+h_{1} u(1)=0
$$

has eigenvalues $\left\{y_{n}\right\}_{n=0}^{\infty}$. (By (1.2) and (A.5c), $h_{1}=-\frac{1}{2} \int_{1 / 2}^{1} d x q(x)$.)

This is just a special case of the construction of Levitan and Gasymov [25] Historically, this classical two-spectra inverse problem goes back to Borg's seminal paper [2]. Subsequently, Levinson [22] found considerable simplifications of Borg's uniqueness arguments, and Krein [20] developed his own solution of these inverse spectral problems. This circle of ideas was further developed in [14, [23, 24], Ch. 3, [25], [26], Sect. 6.11, and continues to generate interest (see, e.g., [17], [27], [36]).

We also need the elementary

Lemma 3.2. Suppose that $h_{0}, h_{1}$, and $q$ are given, and for some $\hat{\lambda}$, there exists an $h_{1 / 2}$ (with the Dirichlet boundary condition $h_{1 / 2}=\infty$ at $x=\frac{1}{2}$ allowed; $u^{\prime}\left(\frac{1}{2}\right)+$ $h_{1 / 2} u\left(\frac{1}{2}\right)=0$ is then interpreted as $\left.u\left(\frac{1}{2}\right)=0\right)$ so that $\hat{\lambda}$ is an eigenvalue of both

$$
-\frac{d^{2}}{d x^{2}}+q \quad \text { in } L^{2}\left(\left(0, \frac{1}{2}\right)\right) ; \quad u^{\prime}(0)+h_{0} u(0)=0, u^{\prime}\left(\frac{1}{2}\right)+h_{1 / 2} u\left(\frac{1}{2}\right)=0
$$

and

$$
-\frac{d^{2}}{d x^{2}}+q \quad \text { in } L^{2}\left(\left(\frac{1}{2}, 1\right)\right) ; \quad u^{\prime}\left(\frac{1}{2}\right)+h_{1 / 2} u\left(\frac{1}{2}\right)=0, u^{\prime}(1)+h_{1} u(1)=0 .
$$


Then $\hat{\lambda}$ is also an eigenvalue of

$$
-\frac{d^{2}}{d x^{2}}+q \quad \text { in } L^{2}((0,1)) ; \quad u^{\prime}(0)+h_{0} u(0)=0, u^{\prime}(1)+h_{1} u(1)=0 .
$$

Proof. One can match the solutions in the two halves so that they and their first derivatives become absolutely continuous near $x=\frac{1}{2}$.

Let $x_{n}^{(0)}=[(2 n) \pi]^{2}$ and $y_{n}^{(0)}=[(2 n+1) \pi]^{2}, n=0,1, \ldots$, be the eigenvalues that lead to $q=0$ on $\left[\frac{1}{2}, 1\right]$ ( and $h_{1}^{(0)}=0$ ) in Proposition 3.1. To construct our example, we will take $x_{n}=x_{n}^{(0)}$ for all $n=0,1, \ldots$ and $y_{n}=y_{n}^{(0)}$ for $n=0,1 \ldots$ with $k+1$ exceptions, say, $y_{n_{0}} \neq y_{n_{0}}^{(0)}, y_{n_{1}} \neq y_{n_{1}}^{(0)}, \ldots, y_{n_{k}} \neq y_{n_{k}}^{(0)}$. Make the choice so that for $\ell=1,2, \ldots, k$,

$$
\sum_{j=0}^{k} y_{n_{j}}^{\ell}=\sum_{j=0}^{k}\left[y_{n_{j}}^{(0)}\right]^{\ell} .
$$

Choices satisfying (3.4) can certainly be made. For example, we can take $y_{n_{0}}=$ $y_{n_{0}}^{(0)}+\varepsilon$ with $\varepsilon$ small, and solve the $k$ equations (3.4) for $y_{n_{1}}, \ldots, y_{n_{k}}$ using the fact that the Jacobian determinant that needs to be non-zero to apply the inverse function theorem is essentially just a $k \times k$ Vandermonde determinant, $\operatorname{det}(a)$, with $a_{j \ell}=\ell\left(y_{n_{j}}^{(0)}\right)^{\ell-1}$.

Let $m_{N,+}^{(0)}\left(z, \frac{1}{2}\right)=-\frac{1}{\sqrt{z}} \cot \left(\frac{1}{2} \sqrt{z}\right)$, the Neumann $m_{+}$-function on $\left[\frac{1}{2}, 1\right]$ for $q=0$ (and $h_{1}^{(0)}=0$ ). Let $m_{N,+}(z)$ be the corresponding Neumann $m_{+}$-function on $\left[\frac{1}{2}, 1\right]$ for the $q$ constructed in Proposition 3.1 (whose poles and zeros are given by the eigenvalues $x_{n}$ and $y_{n}, n=0,1 \ldots$, of (3.2) and (3.3)). We claim that

$$
m_{N,+}\left(z, \frac{1}{2}\right)=\prod_{j=0}^{k}\left(\frac{z-y_{n_{j}}}{z-y_{n_{j}}^{(0)}}\right) m_{N,+}^{(0)}\left(z, \frac{1}{2}\right) .
$$

Indeed, the two sides have the same zeros and poles, and both are ratios of functions of order $\frac{1}{2}$; thus they are constant multiples of each other. Since both sides behave asymptotically like $\sim-|z|^{-1 / 2}$ as $z \rightarrow-\infty$, the constant multiple must be 1 .

Because of (3.4),

$$
\ln \left(\prod_{j=0}^{k}\left(\frac{z-y_{n_{j}}}{z-y_{n_{j}}^{(0)}}\right)\right)=\sum_{j=1}^{k}\left[\ln \left(1-\frac{y_{n_{j}}}{z}\right)-\ln \left(1-\frac{y_{n_{j}}^{(0)}}{z}\right)\right]=O\left(z^{-k-1}\right) .
$$

Since $m_{N,+}^{(0)}\left(z, \frac{1}{2}\right)=-\frac{i}{\sqrt{z}}\left(1+o\left(z^{-K}\right)\right)$ for all $K$ consistent with $q=0$ in $(2.10)$, (3.5) and (3.6) imply that

$$
m_{N,+}\left(z, \frac{1}{2}\right)=-\frac{i}{\sqrt{z}}\left(1+O\left(z^{-k-1}\right)\right) .
$$

Thus in (2.6), $D_{\ell}\left(\frac{1}{2}\right)=0$ for $\ell=1,2, \ldots, 2 k+1$. But by (2.10) and induction, this implies that

$$
q^{(m)}\left(\frac{1}{2}\right)=0 \quad \text { for } m=0,1,2, \ldots, 2 k-1 .
$$

Next, let $q(x)$ be defined a.e. on $[0,1]$ by

$$
\begin{aligned}
q(x) & =0, & & 0 \leq x<\frac{1}{2}, \\
& =\text { constructed } q \uparrow\left(\frac{1}{2}, 1\right), & & \frac{1}{2}<x \leq 1 .
\end{aligned}
$$


By (3.7), $q$ is $C^{2 k-1}$ at $x=\frac{1}{2}$. By Lemma $3.2,-\frac{d^{2}}{d x^{2}}+q$ in $L^{2}((0,1))$, with $u^{\prime}(0)=0$, $u^{\prime}(1)+h_{1} u(1)=0$ boundary conditions, has $\left\{x_{n}^{(0)}\right\}_{n=0}^{\infty} \cup\left\{y_{n}^{(0)}\right\}_{n=0, n \neq n_{0}, n_{1}, \ldots, n_{k}}^{\infty}$ as eigenvalues, so at most $(k+1)$ eigenvalues differ from the free Neumann case (i.e., $q=0$ on $\left.[0,1], h_{0}=h_{1}=0\right)$. If fewer than $k+1$ eigenvalues differed, then by Theorem $1.2, q \equiv 0$ and $h_{1}=0$. Since the $y_{n_{i}}$ are not $y_{n_{i}}^{(0)}$, this cannot be true. Thus, exactly $k+1$ eigenvalues differ. If $q^{(2 k)}\left(\frac{1}{2}\right)$ were zero, then by Theorem 1.2 , again $q \equiv 0$ and $h_{1}=0$, so $q$ is not $C^{2 k}$.

There remains an interesting open question: Can one replace information on the missing eigenvalue by knowledge of the boundary condition $h_{1}$ ?

\section{The Case of Partially Known Spectra}

Our goal in this section is to prove Theorem 1.3. Define

$$
g_{S}(z)=\prod_{\lambda_{n} \in S}\left(1-\frac{z}{\lambda_{n}}\right), \quad g_{\sigma(H)}(z)=\prod_{n=0}^{\infty}\left(1-\frac{z}{\lambda_{n}}\right), \quad S \subseteq \sigma(H)=\left\{\lambda_{n}\right\}_{n=0}^{\infty} .
$$

By the hypothesis (1.4) on $S$ and $\sigma(H)$ in Theorem 1.3 and the method of proof of Theorem B.4 (see the critical equality (B.16)), we infer

$$
\ln \left(\left|g_{S}(i y)\right|\right) \geq(1-\alpha) \ln \left(\left|g_{\sigma(H)}(i y)\right|\right)+\frac{\alpha}{4} \ln \left(1+y^{2}\right)+C_{0} .
$$

Since $\sigma(H)$ is a complete set of eigenvalues for a self-adjoint problem on $[0,1]$, we know that asymptotically

$$
\left.\left|g_{\sigma(H)}(i y)\right| \sim \frac{1}{2}|y|^{1 / 2}\left|e^{\operatorname{Im}}(\sqrt{i})\right| y\right|^{1 / 2} \mid \quad \text { as } y(\text { real }) \rightarrow \infty .
$$

Thus by (4.1), there exists a constant $C>0$ such that

$$
\left|g_{S}(i y)\right| \geq C|y|^{1 / 2}\left|e^{\operatorname{Im}(\sqrt{i})(1-\alpha)|y|^{1 / 2}}\right|
$$

for $|y|$ sufficiently large.

Let $P_{j}(z)=u_{j,+}\left(z, \frac{1}{2}+\frac{\alpha}{2}\right), Q_{j}(z)=u_{j,+}^{\prime}\left(z, \frac{1}{2}+\frac{\alpha}{2}\right), j=1,2$, for the two candidate potentials. Then, since $1-\left(\frac{1}{2}+\frac{\alpha}{2}\right)=\frac{1}{2}(1-\alpha)$, we use (2.3) to infer asymptotically

$$
\left|Q_{j}(i y)\right| \sim \frac{1}{2}|y|^{1 / 2}\left|e^{\operatorname{Im}(\sqrt{i})(1 / 2)(1-\alpha)|y|^{1 / 2}}\right| \quad \text { as } y \text { (real) } \rightarrow \infty .
$$

With (4.2) and (4.3), the arguments in Section 2 extend to prove Theorem 1.3.

\section{Theorems for the Whole Real Line}

Our main goal in this section is to prove Theorem 1.4. So we suppose that $q_{1}(x), q_{2}(x)$ are two potentials on $\mathbb{R}$ satisfying

$$
q_{j}(x) \geq C|x|^{2+\varepsilon}+1, \quad j=1,2,
$$

for some $C, \varepsilon>0$. (The condition in Theorem 1.4 has $-D$ in place of 1 . Just add $D+1$ to initial $q_{j}$ 's to get (5.1) if need be.) Thus for any $z \in \mathbb{C}$, there exist solutions $u_{j, \pm}(x, z)$ of $-u^{\prime \prime}(x)+q_{j}(x) u(x)=z u(x)$ which are $L^{2}$ near $\pm \infty$, and

$$
m_{j, \pm}(z):= \pm \frac{u_{j, \pm}^{\prime}(0, z)}{u_{j, \pm}(0, z)}, \quad j=1,2,
$$

are meromorphic functions of $z$. Let $\left\{\lambda_{m}\right\}_{m=1}^{\infty}$ be the eigenvalues of $-\frac{d^{2}}{d x^{2}}+q_{1}(x)$ on $(-\infty, \infty)$ and denote by $\left\{\mu_{j, m}\right\}_{m=1}^{\infty}$ the eigenvalues of $-\frac{d^{2}}{d x^{2}}+q_{j}(x)$ on $(-\infty, 0]$ with Dirichlet (i.e., $u(0)=0$ ) boundary conditions at $x=0$. We claim: 
Proposition 5.1. (i) Let $\rho_{0}=1-\frac{\varepsilon}{(4+2 \varepsilon)}$. Then for all $\rho>\rho_{0}, j=1,2$,

$$
\begin{array}{r}
\sum_{m=1}^{\infty} \lambda_{m}^{-\rho}<\infty, \\
\sum_{m=1}^{\infty}\left(\mu_{j, m}\right)^{-\rho}<\infty .
\end{array}
$$

(ii) Moreover,

$$
\mu_{j, m} \geq \lambda_{2 m}, \quad m \in \mathbb{N}
$$

Proof. Let $\alpha_{m}$ be the $m^{\text {th }}$ eigenvalue of $-\frac{d^{2}}{d x^{2}}+C x^{2+\varepsilon}$ on $(-\infty, \infty)$. The large $m$ asymptotics of $\alpha_{m}$ is given by a classical phase space argument (see, e.g., Theorem XIII.81 in [32, or Section 7.1 in [38]); that is, for an explicit constant $K \in(0, \infty), \lim _{m \rightarrow \infty} \alpha_{m} / m^{1 / \rho_{0}}=K$. Thus, $\sum_{m \in \mathbb{N}} \alpha_{m}^{-\rho}<\infty$ if $\rho>\rho_{0}$, and so (5.2) holds since $\lambda_{m} \geq \alpha_{m}$. Let $\beta_{j, m}$ be the $m^{\text {th }}$ eigenvalue of $-\frac{d^{2}}{d x^{2}}+q_{j}(-|x|)$ on $(-\infty, \infty)$. By the hypothesis $q_{j}(-|x|) \geq q_{j}(x)$, we infer that $\beta_{j, m} \geq \lambda_{m}$. But by Dirichlet-Neumann alternation, $\mu_{j, m}=\beta_{j, 2 m}$ for $m=1,2, \ldots$, proving (5.4). (5.3) then follows from (5.4).

Define

$$
\begin{aligned}
Q_{j}(z) & =\prod_{m=1}^{\infty}\left(1-\frac{z}{\mu_{j, m}}\right), \\
g(z) & =\prod_{m=1}^{\infty}\left(1-\frac{z}{\lambda_{m}}\right) .
\end{aligned}
$$

Proposition 5.2. For all $y \in \mathbb{R}$,

$$
\frac{\left|Q_{1}(i y) Q_{2}(i y)\right|}{|g(i y)|} \leq 1
$$

Proof. $\left|1-\frac{i y}{w}\right|=\left(1+\frac{y^{2}}{w^{2}}\right)^{1 / 2}$, for $y, w$ real, is monotone decreasing in $w$, so by (5.4),

$$
\left|1-\frac{i y}{\mu_{j, m}}\right| \leq\left|1-\frac{i y}{\lambda_{2 m}}\right| \leq\left|1-\frac{i y}{\lambda_{2 m-1}}\right| .
$$

It follows that

$$
\left|Q_{j}(i y)\right| \leq \prod_{m=1}^{\infty}\left|1-\frac{i y}{\lambda_{2 m}}\right| \leq \prod_{m=1}^{\infty}\left|1-\frac{i y}{\lambda_{2 m-1}}\right|
$$

so (5.5) holds.

Now let

$$
P_{j}(z)=m_{j,-}(z) Q_{j}(z), \quad j=1,2,
$$

which are entire functions, and

$$
f_{j}(z)=m_{j,-}(z), \quad j=1,2 .
$$

Define

$$
F(z)=\frac{P_{1}(z) Q_{2}(z)-Q_{1}(z) P_{1}(z)}{g(z)}=\frac{Q_{1}(z) Q_{2}(z)}{g(z)}\left(f_{1}(z)-f_{2}(z)\right) .
$$


Proof of Theorem 1.4. At $z=\lambda_{k}$, the eigenfunctions on the left half-line for both $q_{1}$ and $q_{2}$ must match to the common eigenfunctions on the right, so $P_{1}\left(\lambda_{k}\right) Q_{2}\left(\lambda_{k}\right)-$ $P_{2}\left(\lambda_{k}\right) Q_{1}\left(\lambda_{k}\right)=0$; that is, $F(z)$ is an entire function.

By (5.2), $g(z)$ is a function of $m$-type as defined in Appendix B. Thus by Proposition B.5, there exists a sequence $R_{k} \rightarrow \infty$ so that $\sup \left\{\frac{1}{|g(z)|}|| z \mid=R_{k}\right\} \leq$ $C_{1} \exp \left(+C_{2} R_{k}^{\rho}\right)$ for some $\rho<1$. By $(5.3)$ and a similar estimate for the Dirichlet eigenvalues, $P_{j}, Q_{j}$ are functions of $m$-type. It follows by Proposition B.6 that if

$$
\lim _{|y| \rightarrow \infty}|F(i y)|=0,
$$

then $F \equiv 0$. If we prove that, then $m_{1,-}(z)=m_{2,-}(z)$, and thus $q_{1}=q_{2}$ a.e. on $(-\infty, 0]$ and hence on $\mathbb{R}$. Thus, we need only prove (5.7).

By (2.4), which holds for half-line $m$-functions [1], 8], $\left|f_{1}(i y)-f_{2}(i y)\right|=o(1)$. Thus, Proposition 5.2 and (5.6) show that (5.7) holds.

Several questions remain open. We do not believe that hypothesis (i) is needed in Theorem 1.4:

Conjecture 5.3. Theorem 1.4 remains true if (i) is replaced by $\lim _{|x| \rightarrow \infty} q(x)=$ $\infty$.

This will require dealing with entire functions of order larger than 1 .

We also believe:

\section{Conjecture 5.4. Suppose}

$$
\lim _{x \rightarrow \infty} \frac{q(-x)}{q(x)}=\infty \quad \text { and } \quad \lim _{|x| \rightarrow \infty} q(x)=\infty .
$$

Then $q$ near $+\infty$ and a finite density subset of eigenvalues for $-\frac{d^{2}}{d x^{2}}+q(x)$ uniquely determine $q$ on $\mathbb{R}$.

\section{ACKNOWLEDGMENTS}

We would like to thank R. del Rio for discussions and pertinent hints to the literature. F.G. is indebted to A.S. Kechris and C.W. Peck for a kind invitation to Caltech during the summer of 1996, where some of this work was done. The extraordinary hospitality and support by the Department of Mathematics at Caltech are gratefully acknowledged. B.S. would like to thank M. Ben-Artzi and the Hebrew University, where some of this work was done during a visit.

\section{Appendix A: Dirichlet Boundary Conditions}

In this appendix we provide some details in the remaining cases, which involve Dirichlet boundary conditions at $x=0$ and/or $x=1$. We need to distinguish three cases (cf. (1.1)):

(I) $H$ has a Dirichlet boundary condition at $x=0$ and $x=1$, that is,

$$
\begin{aligned}
& u(0)=0, \\
& u(1)=0 .
\end{aligned}
$$


(II) $H$ has a non-Dirichlet boundary condition at $x=0$ and a Dirichlet boundary condition at $x=1$, that is,

$$
\begin{aligned}
u^{\prime}(0)+h_{0} u(0) & =0, \quad h_{0} \in \mathbb{R}, \\
u(1) & =0 .
\end{aligned}
$$

(III) $H$ has a Dirichlet boundary condition at $x=0$ and a non-Dirichlet boundary condition at $x=1$, that is,

$$
\begin{aligned}
u(0) & =0, \\
u^{\prime}(1)+h_{1} u(1) & =0, \quad h_{1} \in \mathbb{R} .
\end{aligned}
$$

Since, later on, $q$ is supposed to be known on $\left[0, \frac{1}{2}+\varepsilon\right]$ for some $0 \leq \varepsilon<\frac{1}{2}$, cases II and III represent inequivalent situations and need to be treated separately in connection with Theorems A.1-A.3. Depending on the case at hand, we index the corresponding eigenvalues $\lambda_{n}$ of $H$ by

$$
\left\{\lambda_{n}\right\}_{n=1}^{\infty} \text { in case I and }\left\{\lambda_{n}\right\}_{n=0}^{\infty} \text { in cases II and III. }
$$

The asymptotic expansion (1.2) then becomes, as $n \rightarrow \infty$,

$$
\begin{array}{ll}
\lambda_{n}=(\pi n)^{2}+\int_{0}^{1} d x q(x)+o(1) & \text { in case I, } \\
\lambda_{n}=\left(\pi\left(n+\frac{1}{2}\right)\right)^{2}-2 h_{0}+\int_{0}^{1} d x q(x)+o(1) & \text { in case II, } \\
\lambda_{n}=\left(\pi\left(n+\frac{1}{2}\right)\right)^{2}+2 h_{1}+\int_{0}^{1} d x q(x)+o(1) & \text { in case III. }
\end{array}
$$

Let $u_{+}(z, x)$ be defined by $-u_{+}^{\prime \prime}+q u_{+}=z u_{+}$subject to the boundary conditions and normalizations

$$
\begin{array}{lll}
u_{+}^{\prime}(z, 1)=1, & u_{+}(z, 1)=0 & \text { in cases I and II, } \\
u_{+}^{\prime}(z, 1)=-h_{1}, & u_{+}(z, 1)=1 & \text { in case III. }
\end{array}
$$

Next we note that items (1) and (3)-(5) of Section 2 remain valid in the present cases I-III, whereas item (2) becomes

$$
\begin{aligned}
& u_{+}(z, x)=-\frac{\sin (\sqrt{z}(1-x))}{\sqrt{z}}+O\left(\frac{e^{\operatorname{Im}(\sqrt{z})(1-x)}}{z}\right), \\
& u_{+}^{\prime}(z, x)=\cos (\sqrt{z}(1-x))+O\left(\frac{e^{\operatorname{Im}(\sqrt{z})(1-x)}}{\sqrt{z}}\right)
\end{aligned}
$$

in cases I and II, and

$$
\begin{aligned}
& u_{+}(z, x)=\cos (\sqrt{z}(1-x))+O\left(\frac{e^{\operatorname{Im}(\sqrt{z})(1-x)}}{\sqrt{z}}\right), \\
& u_{+}^{\prime}(z, x)=\sqrt{z} \sin (\sqrt{z}(1-x))+O\left(e^{\operatorname{Im}(\sqrt{z})(1-x)}\right)
\end{aligned}
$$

in case III, as $|z| \rightarrow \infty$ for all $x \in[0,1]$.

Introducing $P_{j}, Q_{j}, f_{j}, j=1,2$, as in (2.11)-(2.13), and $g$ by

$$
\begin{array}{ll}
g(z)=u_{1,+}(z, 0) & \text { in cases I and III, } \\
g(z)=u_{1,+}^{\prime}(z, 0)+h_{0} u_{1,+}(z, 0) & \text { in case II, }
\end{array}
$$


one infers again that $\left\{\lambda_{1, n}\right\}_{n=1}^{\infty}$ in case I and $\left\{\lambda_{1, n}\right\}_{n=0}^{\infty}$ in cases II and III are precisely the zeros of $g(z)$. The corresponding properties (a)-(g) listed in Section 2, suitably adapted to the present cases I-III, then read as follows:

$\left(\mathrm{a}^{\prime}\right)$ Item (a) remains valid except that $n$ in (2.15) runs through $\mathbb{N}$ in case I and through $\mathbb{N}_{0}$ in cases II and III.

$\left(b^{\prime}-d^{\prime}\right)$ Items $(b),(c)$, and $(d)$ remain valid.

$\left(\mathrm{e}^{\prime}\right)$ As $y($ real $) \rightarrow \infty$,

$$
\begin{aligned}
& \left|Q_{j}(i y)\right|=\frac{1}{2}\left|\exp \left(\frac{1}{2} \operatorname{Im}(\sqrt{i})|y|^{1 / 2}\right)\right|(1+o(1)) \quad \text { in cases I and II, } \\
& \left|Q_{j}(i y)\right|=\frac{1}{2}|y|^{1 / 2}\left|\exp \left(\frac{1}{2} \operatorname{Im}(\sqrt{i})|y|^{1 / 2}\right)\right|(1+o(1)) \quad \text { in case III. }
\end{aligned}
$$

$\left(\mathrm{f}^{\prime}\right)$ As $y($ real $) \rightarrow \infty$,

$$
\begin{aligned}
& |g(i y)|=\frac{1}{2}|y|^{-1 / 2}\left|\exp \left(\operatorname{Im}(\sqrt{i})|y|^{1 / 2}\right)\right|(1+o(1)) \quad \text { in case I, } \\
& |g(i y)|=\frac{1}{2}\left|\exp \left(\operatorname{Im}(\sqrt{i})|y|^{1 / 2}\right)\right|(1+o(1)) \quad \text { in cases II and III. }
\end{aligned}
$$

$\left(\mathrm{g}^{\prime}\right)$ For $n$ sufficiently large, one obtains,

$$
\begin{array}{ll}
\inf _{\theta \in[0,2 \pi]}\left|g\left(\left(\pi\left(n+\frac{1}{2}\right)\right)^{2} e^{i \theta}\right)\right| \geq \frac{1}{\pi n}+O\left(\frac{1}{n^{2}}\right) & \text { in case I, } \\
\inf _{\theta \in[0,2 \pi]}\left|g\left((\pi(n+1))^{2} e^{i \theta}\right)\right| \geq 1+O\left(\frac{1}{n}\right) & \text { in cases II and III. }
\end{array}
$$

Introducing $F(z)$ as in (2.16) and (2.18), one verifies, using $\left(\mathrm{b}^{\prime}\right)$ and $\left(\mathrm{g}^{\prime}\right)$, that (2.17) remains valid. Items $\left(\mathrm{c}^{\prime}\right),\left(\mathrm{e}^{\prime}\right)$, and $\left(\mathrm{f}^{\prime}\right)$, however, yield the following modification of $(2.19)$ as $y($ real $) \rightarrow \infty$,

$$
\begin{aligned}
& |F(i y)|=\frac{1}{2}|y|^{1 / 2}\left[o\left(|y|^{-1}\right)\right](1+o(1))=o\left(|y|^{-1 / 2}\right) \quad \text { in case I, } \\
& |F(i y)|=\frac{1}{2}\left[o\left(|y|^{-1}\right)\right](1+o(1))=o\left(|y|^{-1}\right) \quad \text { in case II, } \\
& |F(i y)|=\frac{1}{2}|y|\left[o\left(|y|^{-1}\right)\right](1+o(1))=o(1) \quad \text { in case III. }
\end{aligned}
$$

Following the arguments in (2.16)-(2.19) step-by-step and taking into account the eigenvalue asymptotics in (A.5), the remaining Dirichlet cases in Theorem 1.1 then read as follows.

Theorem A.1. Let $H=-\frac{d^{2}}{d x^{2}}+q$ in $L^{2}((0,1))$ with boundary conditions (A.1), (A.2), or (A.3) for cases I, II, or III. Then $q$ on $\left[0, \frac{1}{2}\right]$, together with the knowledge of $h_{0}=\infty$ or $h_{0} \in \mathbb{R}$, and all the eigenvalues of $H$, uniquely determine $h_{1}$ (i.e., $h_{1}=\infty$ in cases I and II and $h_{1} \in \mathbb{R}$ in case III) and $q$ (a.e.) on all of $[0,1]$.

Remark. Case I for $q \in L^{2}((0,1))$ appears to be due to Pöschel and Trubowitz [31], Ch. 4. Much to our surprise, the extension of case I to $q \in L^{1}((0,1))$ in Theorem A.1 seems to be new. Case II is originally due to Hochstadt and Lieberman [16] as recorded in Theorem 1.1. To the best of our knowledge, case III is a new result.

The analog of Theorem 1.2 is now obtained as follows. Replace the definition of $\tilde{F}$ in $(2.20)$ by

$$
\begin{aligned}
& \tilde{F}(z)=\frac{\left[P_{1}(z) Q_{2}(z)-P_{2}(z) Q_{1}(z)\right]}{g(z)} \prod_{\ell=1}^{k+1}\left(z-\lambda_{\ell}\right) \quad \text { in cases I and II, } \\
& \tilde{F}(z)=\frac{\left[P_{1}(z) Q_{2}(z)-P_{2}(z) Q_{1}(z)\right]}{g(z)} \prod_{\ell=1}^{k}\left(z-\lambda_{\ell}\right) \quad \text { in case III. }
\end{aligned}
$$


Here $\left\{\lambda_{\ell}\right\}_{\ell=1}^{k+1}$ in cases I and II, and $\left\{\lambda_{\ell}\right\}_{\ell=1}^{k}$ in case III, represent eigenvalues of $H_{1}$ and $H_{2}$ which are not a priori assumed to be equal.

The asymptotic behavior of $|\tilde{F}(i y)|$ as $y$ (real) $\rightarrow \infty$,

$$
\begin{aligned}
|\tilde{F}(i y)| & =o(1) & & \text { in case I, } \\
|\tilde{F}(i y)| & =o\left(|y|^{-1 / 2}\right) & & \text { in cases II and III, }
\end{aligned}
$$

then yields the following new result.

Theorem A.2. Let $H=-\frac{d^{2}}{d x^{2}}+q$ in $L^{2}((0,1))$ with boundary conditions (A.1), (A.2), or (A.3) for cases I, II, or III. Suppose $q$ is $C^{2 k}\left(\left(\frac{1}{2}-\varepsilon, \frac{1}{2}+\varepsilon\right)\right), k=0,1, \ldots$, for some $\varepsilon>0$. Then $q$ on $\left[0, \frac{1}{2}\right]$, together with the knowledge of $h_{0}=\infty$ or $h_{0} \in \mathbb{R}$, and all the eigenvalues of $H$ except for $(k+1)$ in cases I and II and $k$ in case III, uniquely determine $h_{1}$ (i.e., $h_{1}=\infty$ in cases I and II and $h_{1} \in \mathbb{R}$ in case III) and $q$ on all of $[0,1]$.

Finally, we consider the analog of Theorem 1.3 in the Dirichlet context.

Theorem A.3. Let $H=-\frac{d^{2}}{d x^{2}}+q$ in $L^{2}((0,1))$ with boundary conditions (A.1), (A.2), or (A.3). Then $q$ on $\left[0, \frac{1}{2}+\frac{\alpha}{2}\right]$ for some $\alpha \in(0,1), h_{0}=\infty$ or $h_{0} \in \mathbb{R}$, and a subset $S \subseteq \sigma(H)$ of all the eigenvalues $\sigma(H)$ of $H$ satisfying

$$
\#\left\{\lambda \in S \mid \lambda \leq \lambda_{0}\right\} \geq(1-\alpha) \#\left\{\lambda \in \sigma(H) \mid \lambda \leq \lambda_{0}\right\}+\frac{\alpha}{2}
$$

for all sufficiently large $\lambda_{0} \in \mathbb{R}$, uniquely determine $h_{1}$ (i.e., $h_{1}=\infty$ in cases $\mathrm{I}$ and II and $h_{1} \in \mathbb{R}$ in case III) and $q$ on all of $[0,1]$.

Proof. Following the arguments employed in Section 4, we again introduce

$$
\begin{aligned}
g_{S}(z)=\prod_{\lambda_{n} \in S}\left(1-\frac{z}{\lambda_{n}}\right), & S \subseteq \sigma(H), \\
g_{\sigma(H)}(z)=\prod_{n=1}^{\infty}\left(1-\frac{z}{\lambda_{n}}\right) & \text { in case I, } \\
g_{\sigma(H)}(z)=\prod_{n=0}^{\infty}\left(1-\frac{z}{\lambda_{n}}\right) & \text { in cases II and III, }
\end{aligned}
$$

where

$$
\sigma(H)=\left\{\lambda_{n}\right\}_{n=1}^{\infty} \quad \text { in case I } \quad \text { and } \quad \sigma(H)=\left\{\lambda_{n}\right\}_{n=0}^{\infty} \quad \text { in cases II and III. }
$$

Then (A.15) and the method of proof of Theorem B.4 yield

$$
\ln \left(\left|g_{S}(i y)\right|\right) \geq(1-\alpha) \ln \left(\left|g_{\sigma(H)}(i y)\right|\right)+\frac{\alpha}{4} \ln \left(1+y^{2}\right)+C_{0} .
$$

Since asymptotically (cf. $\left.\left(\mathrm{f}^{\prime}\right)\right)$ for $|y|$ large enough,

$$
\begin{aligned}
\left|g_{\sigma(H)}(i y)\right| \sim \frac{1}{2}|y|^{-1 / 2}\left|e^{\operatorname{Im}(\sqrt{i})|y|^{1 / 2}}\right| & & \text { in case I, } \\
\left|g_{\sigma(H)}(i y)\right| \sim \frac{1}{2}\left|e^{\operatorname{Im}(\sqrt{i})|y|^{1 / 2}}\right| & & \text { in cases II and III, }
\end{aligned}
$$

one infers from (A.16) that for some $C>0$

$$
\begin{aligned}
& \left|g_{S}(i y)\right| \geq C|y|^{-1 / 2}\left|e^{\operatorname{Im}(\sqrt{i})(1-\alpha)|y|^{1 / 2}}\right| \text { in case I, } \\
& \left|g_{S}(i y)\right| \geq C\left|e^{\operatorname{Im}(\sqrt{i})(1-\alpha)|y|^{1 / 2}}\right| \quad \text { in cases II and III }
\end{aligned}
$$

for $|y|$ sufficiently large. 
Introducing again $P_{j}(z)=u_{j,+}\left(z, \frac{1}{2}+\frac{\alpha}{2}\right), Q_{j}(z)=u_{j,+}^{\prime}\left(z, \frac{1}{2}+\frac{\alpha}{2}\right), j=1,2$, for the two candidate potentials, noticing that $1-\left(\frac{1}{2}+\frac{\alpha}{2}\right)=\frac{1}{2}(1-\alpha)$, we infer asymptotically that as $y$ (real) $\rightarrow \infty$,

$$
\begin{array}{rlrl}
\left|Q_{j}(i y)\right| \sim \frac{1}{2}\left|e^{\operatorname{Im}(\sqrt{i})(1 / 2)(1-\alpha)|y|^{1 / 2}}\right| & & \text { in cases I and II, } \\
\left|Q_{j}(i y)\right| \sim \frac{1}{2}|y|^{1 / 2}\left|e^{\operatorname{Im}(\sqrt{i})(1 / 2)(1-\alpha)|y|^{1 / 2}}\right| & \text { in case III. }
\end{array}
$$

Given (A.17) and (A.18), one can now finish the proof of Theorem A.3 in the same way as that of Theorem 1.3 in Section 4.

Remark. As in the case $\alpha=0$, we have an extension of the same type as Theorem A.2. Explicitly, if $q$ is assumed to be $C^{2 k}$ near $x=\frac{1}{2}+\frac{\alpha}{2}$, we only need

$$
\#\left\{\lambda \in S \mid \lambda \leq \lambda_{0}\right\} \geq(1-\alpha) \#\left\{\lambda \in \sigma(H) \mid \lambda \leq \lambda_{0}\right\}+\frac{\alpha}{2}-N(k)
$$

instead of (A.15), where $N(k)=k+1$ in cases I and II and $N(k)=k$ in case III.

\section{Appendix B: Zeros of Entire Functions}

In discussing extensions of Hochstadt's discrete (finite matrix) version [15] of the Hochstadt-Lieberman theorem in [12], we made use of the following simple lemma, which is an elementary consequence of the fact that any polynomial of degree $d$ with $d+1$ zeros must be the zero polynomial:

Lemma B.1. Suppose $f_{1}=\frac{P_{1}}{Q_{1}}$ and $f_{2}=\frac{P_{2}}{Q_{2}}$ are two rational fractions where the polynomials satisfy $\operatorname{deg}\left(P_{1}\right)=\operatorname{deg}\left(P_{2}\right)$ and $\operatorname{deg}\left(Q_{1}\right)=\operatorname{deg}\left(Q_{2}\right)$. Suppose that $d=$ $\operatorname{deg}\left(P_{1}\right)+\operatorname{deg}\left(Q_{1}\right)$ and that $f_{1}\left(z_{n}\right)=f_{2}\left(z_{n}\right)$ for $d+1$ distinct points $\left\{z_{n}\right\}_{n=1}^{d+1} \in \mathbb{C}$. Then $f_{1}=f_{2}$.

Our main goal in this appendix is to prove an analogous theorem for a class of entire functions.. The theorem is sharp in the sense that it includes Lemma B.1 (at least the case of Lemma B.1 where the zeros of the entire functions involved and the $z_{n}$ are all positive).

We will be interested here in entire functions of the form

$$
f(z)=C \prod_{n=0}^{\infty}\left(1-\frac{z}{x_{n}}\right)
$$

where $0<x_{0}<x_{1}<\cdots$ is a suitable sequence of positive numbers which are the zeros of $f$, and $C$ is some complex constant.

Given a sequence $\left\{x_{n}\right\}_{n=0}^{\infty}$ of positive reals, define

$$
N(t)=\#\left\{n \in \mathbb{N} \cup\{0\} \mid x_{n}<t\right\} .
$$

Recall the following basic theorem (see, e.g., [21, Ch. I; [30], Sects. II.48 and II.49):

Theorem B.2. Fix $0<\rho_{0}<1$. Then:

(i) If $\left\{x_{n}\right\}_{n=0}^{\infty}$ is a sequence of positive reals with

$$
\sum_{n=0}^{\infty} x_{n}^{-\rho}<\infty \quad \text { for all } \rho>\rho_{0}
$$

then the product in (B.1) defines an entire function $f$ with

$$
|f(z)| \leq C_{1} \exp \left(C_{2}|z|^{\rho}\right) \quad \text { for all } \rho>\rho_{0} .
$$


(ii) Conversely, if $f$ is an entire function satisfying (B.4) with all its (complex) zeros on $(0, \infty)$, then its zeros $\left\{x_{n}\right\}_{n=0}^{\infty}$ satisfy (B.3), and $f$ has the canonical product expansion (B.1).

Moreover, (B.3) holds if and only if

$$
N(t) \leq C|t|^{\rho} \quad \text { for all } \rho>\rho_{0} .
$$

Given this theorem, we single out:

Definition B.3. A function $f$ is said to be of $m$-type if and only if $f$ is an entire function satisfying (B.4) (of order $0<\rho<1$ in the usual definition) with all the zeros of $f$ on $(0, \infty)$.

Our choice of " $m$-type" in Definition B.3 comes from the fact that in many cases we discuss in this paper, the $m$-function is a ratio of functions of $m$-type. By Theorem B.2, $f$ in Definition B.3 has the form (B.1) and $N(t)$, which we will denote as $N_{f}(t)$, satisfies (B.5). We are heading toward a proof of

Theorem B.4. Let $f_{1}, f_{2}, g$ be three functions of $m$-type so that

(i) $f_{1}(z)=f_{2}(z)$ at any point $z$ with $g(z)=0$, and

(ii) for all sufficiently large $t$,

$$
\max \left(N_{f_{1}}(t), N_{f_{2}}(t)\right) \leq N_{g}(t)-1
$$

Then $f_{1}=f_{2}$.

Proposition B.5. Let $f$ be a function of $m$-type. Then there exists a $0<\rho<1$ and a sequence $\left\{R_{k}\right\}_{k=1}^{\infty}, R_{k} \rightarrow \infty$ as $k \rightarrow \infty$, so that

$$
\inf \left\{|f(z)||| z \mid=R_{k}\right\} \geq C_{1} \exp \left(-C_{2} R_{k}^{\rho}\right) .
$$

Proof. By hypothesis, for some $0<\rho^{\prime}<1$,

$$
N_{f}(t) \leq C t^{\rho^{\prime}}
$$

This implies

$$
x_{n} \geq\left(\frac{n}{C}\right)^{1 / \rho^{\prime}}
$$

If for all $n \geq n_{0}$,

$$
\left|x_{n}-x_{n-1}\right| \leq 2
$$

then

$$
x_{n} \leq x_{n_{0}}+2\left(n-n_{0}\right),
$$

which contradicts (B.7). Thus for an infinite sequence $\{n(k)\}_{k=1}^{\infty}, n(k) \rightarrow \infty$ as $k \rightarrow \infty$, we necessarily must have

$$
x_{n(k)}-x_{n(k)-1} \geq 2 .
$$

We will pick

$$
R_{k}=\frac{1}{2}\left(x_{n(k)}+x_{n(k)-1}\right) .
$$

For any $\alpha>0,\left|1-\alpha e^{i \theta}\right|^{2}=1+\alpha^{2}-2 \alpha \cos (\theta)$ takes its minimum value at $\theta=0$, so

$$
\inf \left\{|f(z)| \mid z=R_{k}\right\}=\left|f\left(R_{k}\right)\right|
$$


By Theorem B.2, $f$ has the form (B.1). We will write

$$
\ln \left(\left|f\left(R_{k}\right)\right|\right)=A_{1}+A_{2},
$$

where

$$
A_{1}=\sum_{n \mid x_{n} \geq 2 x_{n(k)}} \ln \left(\left|1-\frac{R_{k}}{x_{n}}\right|\right), \quad A_{2}=\sum_{n \mid x_{n}<2 x_{n(k)}} \ln \left(\left|1-\frac{R_{k}}{x_{n}}\right|\right) .
$$

We estimate $A_{1}$ by writing the sum as a Stieltjes integral, integrating by parts, and using (B.6):

$$
\begin{aligned}
A_{1} & =\int_{2 x_{n(k)}}^{\infty} \ln \left(1-\frac{R_{k}}{t}\right) d N_{f}(t) \\
& =-\int_{2 x_{n(k)}}^{\infty}\left(\frac{1}{1-\frac{R_{k}}{t}}\right) \frac{R_{k}}{t^{2}}\left[N_{f}(t)-N_{f}\left(2 x_{n(k)}\right)\right] d t \\
& \geq-\int_{2 R_{k}}^{\infty} \frac{2 R_{k}}{t^{2}} C t^{\rho^{\prime}} d t=-C R_{k}^{\rho^{\prime}},
\end{aligned}
$$

where we have used $C$ to represent a positive constant that varies from formula to formula.

For $A_{2}$, we write,

$$
\begin{aligned}
\ln \left(\left|1-\frac{R_{k}}{x_{n}}\right|\right) & =\ln \left(\left|x_{n}-R_{k}\right|\right)-\ln \left(\left|x_{n}\right|\right) \geq-\ln \left(\left|x_{n}\right|\right) \\
& \geq-\ln \left(4 R_{k}\right),
\end{aligned}
$$

where (B.13) follows from (B.8) and (B.9), and (B.14) follows from

$$
\left|x_{n}\right|<2 x_{n(k)} \leq 2\left(2 R_{k}\right) \text {. }
$$

Thus, by (B.6),

$$
A_{2} \geq-N_{f}\left(2 x_{n(k)}\right) \ln \left(4 R_{k}\right) \geq-C R_{k}^{\rho^{\prime}} \ln \left(4 R_{k}\right) \geq-C R_{k}^{\rho^{\prime \prime}}
$$

for some $1>\rho^{\prime \prime}>\rho^{\prime}$ and suitable positive constants $C$. (B.10), (B.11), (B.12), and (B.15) prove the proposition.

Proposition B.6. Let $F$ be an entire function that satisfies

(i) $\sup _{|z|=R_{k}}|F(z)| \leq C_{1} \exp \left(C_{2} R_{k}^{\rho}\right)$ for some $0 \leq \rho<1, C_{1}, C_{2}>0$, and some sequence $R_{k} \rightarrow \infty$ as $k \rightarrow \infty$.

(ii) $\lim _{|x| \rightarrow \infty ; x \in \mathbb{R}}|F(i x)|=0$.

Then $F \equiv 0$.

Proof. A standard Phragmén-Lindelöf estimate separately applied to $\operatorname{Re}(z)>0$ and $\operatorname{Re}(z)<0$ (i.e., to an angle of opening $\pi$; see, e.g., 30, Sect. II.34) shows that $F$ is bounded. Liouville's theorem implies that $F$ is constant, and then the fact that $|F(i x)| \rightarrow 0$ as $x \rightarrow \infty$ ( $x$ real) shows that $F=0$.

Proof of Theorem B.4. By hypothesis (i), $\frac{f_{1}(z)-f_{2}(z)}{g(z)}:=Q(z)$ is an entire function. By (B.4) (applied to $f_{1}$ and $f_{2}$ ) and Proposition B.5 (applied to $g$ ), there is a sequence $R_{k} \rightarrow \infty$ as $k \rightarrow \infty$ so that $Q(z)$ satisfies condition (i) of Proposition B.6. Thus, it suffices to prove

$$
\lim _{x \rightarrow \pm \infty} \frac{f_{1}(i x)}{g(i x)}=\lim _{x \rightarrow \pm \infty} \frac{f_{2}(i x)}{g(i x)}=0 .
$$


We will prove the $f_{1}$ result for definiteness. In fact, our proof will show that

$$
\frac{f_{1}(i x)}{g(i x)}=O\left(x^{-1}\right)
$$

as $|x| \rightarrow \infty$. Without loss of generality, we will assume that $f_{1}, g$ satisfy (B.1) with $C=1$, that is, that $f_{1}(0)=g(0)=1$. We will also suppose that $N_{f_{1}}(t)=N_{g}(t)=0$ if $t \leq 1$, which can be arranged by appropriate scaling.

Notice first that

$$
\begin{aligned}
\ln \left(\left|f_{1}(i x)\right|\right) & =\sum_{n=0}^{\infty} \frac{1}{2} \ln \left(1+\frac{x^{2}}{x_{n}^{2}}\right) \\
& =\frac{1}{2} \int_{0}^{\infty} \ln \left(1+\frac{x^{2}}{t^{2}}\right) d N_{f_{1}}(t) \\
& =\frac{1}{2} \int_{0}^{\infty} \frac{1}{1+\frac{x^{2}}{t^{2}}} \frac{2 x^{2}}{t^{3}} N_{f_{1}}(t) d t \\
& =\int_{0}^{\infty} \frac{x^{2}}{t^{3}+t x^{2}} N_{f_{1}}(t) d t .
\end{aligned}
$$

The boundary term at $t=0$ in the integration by parts step vanishes since $N_{f_{1}}(0)=$ 0 , and the one at $t=\infty$ vanishes by the estimate (B.5) and the fact that, for $x$ fixed, $\ln \left(1+\frac{x^{2}}{t^{2}}\right)=O\left(t^{-2}\right)$ as $t \rightarrow \infty$.

By hypothesis (ii) of the theorem, there are $t_{0} \geq 1$ and $C \geq 0$ such that

$$
\begin{aligned}
N_{f_{1}}(t) & \leq N_{g}(t)-1, & & t \geq t_{0}, \\
& \leq N_{g}(t)+C, & & t \leq t_{0} .
\end{aligned}
$$

Hence, by (B.16),

$$
\begin{aligned}
\ln \left(\left|\frac{f_{1}(i x)}{g(i x)}\right|\right) & \leq(C+1) \int_{1}^{t_{0}} \frac{x^{2}}{t^{3}+t x^{2}} d t-\int_{1}^{\infty} \frac{x^{2}}{t^{3}+t x^{2}} d t \\
& \leq(C+1) \ln \left(t_{0}\right)-\frac{1}{2} \ln \left(1+x^{2}\right)
\end{aligned}
$$

since

$$
\frac{x^{2}}{t^{3}+t x^{2}}=-\frac{d}{d t}\left[\frac{1}{2} \ln \left(1+\frac{x^{2}}{t^{2}}\right)\right]
$$

Thus, as claimed,

$$
\frac{f_{1}(i x)}{g(i x)}=O\left(x^{-1}\right)
$$

as $|x| \rightarrow \infty$.

One can replace (B.17a) by the following pair of conditions for $t \geq t_{0} \geq 1, t_{0}$ sufficiently large:

$$
\begin{gathered}
N_{f_{1}}(t) \leq N_{g}(t)+D \quad \text { for some } D>-1, \\
\varlimsup_{t \rightarrow \infty} t^{-(D+1)^{-1}}\left|\left\{s \in\left[t_{0}, t\right] \mid N_{f_{1}}(s)>N_{g}(s)-1\right\}\right|=0,
\end{gathered}
$$

where $|\cdot|$ signifies Lebesgue measure. Indeed, denoting by

$$
\gamma(t)=\left|\left\{s \in\left[t_{0}, t\right] \mid N_{f_{1}}(s)>N_{g}(s)-1\right\}\right|
$$


the Lebesgue measure of the set in (B.19), the method of proof in Theorem B.4, together with (B.18) and (B.19), implies

$$
\begin{aligned}
& \ln \left(\left|\frac{f_{1}(i x)}{g(i x)}\right|\right)=\int_{0}^{\infty} \frac{x^{2}}{t^{3}+t x^{2}}\left[N_{f_{1}}(t)-N_{g}(t)\right] d t \\
& \leq C \int_{1}^{t_{0}} \frac{x^{2}}{t^{3}+t x^{2}} d t+\int_{t_{0}}^{\infty} \frac{x^{2}}{t^{3}+t x^{2}}\left[N_{f_{1}}(t)-N_{g}(t)\right] d t \\
&= C \int_{1}^{t_{0}} \frac{x^{2}}{t^{3}+t x^{2}} d t+\int_{\left\{t \in\left[t_{0}, \infty\right) \mid N_{f_{1}}(t) \leq N_{g}(t)-1\right\}} \frac{x^{2}}{t^{3}+t x^{2}}\left[N_{f_{1}}(t)-N_{g}(t)\right] d t \\
&+\int_{\left\{t \in\left[t_{0}, \infty\right) \mid N_{f_{1}}(t)>N_{g}(t)-1\right\}} \frac{x^{2}}{t^{3}+t x^{2}}\left[N_{f_{1}}(t)-N_{g}(t)\right] d t \\
& \leq C \int_{1}^{t_{0}} \frac{x^{2}}{t^{3}+t x^{2}} d t-\int_{t_{0}}^{\infty} \frac{x^{2}}{t^{3}+t x^{2}} d t \\
&+(D+1) \int_{\left\{t \in\left[t_{0}, \infty\right) \mid N_{f_{1}}(t)>N_{g}(t)-1\right\}} \frac{x^{2}}{t^{3}+t x^{2}} d t \\
& \leq(C+1) \ln \left(t_{0}\right)-\frac{1}{2} \ln \left(1+x^{2}\right) \\
&+(D+1) \int_{x}^{\infty} \frac{x^{2}}{t^{3}+t x^{2}} d t+(D+1) \int_{\left\{t \in[1, x] \mid N_{f_{1}}(t)>N_{g}(t)-1\right\}} \frac{x^{3}+t x^{2}}{d t} \\
& \leq(C+1) \ln \left(t_{0}\right)-\frac{1}{2} \ln \left(1+x^{2}\right)+\frac{1}{2}(D+1) \ln (2) \\
&+(D+1) \int_{1}^{\gamma(x)+1} \frac{x^{2}}{t^{3}+t x^{2}} d t \\
& \leq(C+1) \ln \left(t_{0}\right)-\frac{1}{2} \ln \left(1+x^{2}\right)+\frac{1}{2}(D+1) \ln (2)+(D+1) \int_{1}^{\gamma(x)+1} \frac{d t}{t} \\
& \leq-\frac{1}{2} \ln \left(1+x^{2}\right)+\frac{1}{2}(D+1)\left[\ln (2)+\ln \left((1+\gamma(x))^{2}\right)\right]+(C+1) \ln \left(t_{0}\right) .
\end{aligned}
$$

In particular, (B.19) is precisely the result needed in (B.20) to ensure that the limit of $\left|f_{1}(i x) / g(i x)\right|$ as $|x| \rightarrow \infty$ is zero. In (B.20) we used the obvious inequality $x^{2} /\left[t^{3}+t x^{2}\right]<\left(\frac{1}{t}\right)$ for $t>0$ and the fact that

$$
\int_{\Omega} f(t) d t \leq \int_{1}^{|\Omega|+1} f(t) d t
$$

whenever $\Omega \subseteq[1, \infty)$ has finite Lebesgue measure, $|\Omega|<\infty$, and $f$ is monotone decreasing on $[1, \infty)$.

An interesting case is $D=0$ in (B.18)-(B.20).

\section{REFERENCES}

[1] F.V. Atkinson, On the location of the Weyl Circles, Proc. Roy. Soc. Edinburgh 88A 1981 345-356 MR 83a:34023

[2] G. Borg, Eine Umkehrung der Sturm-Liouvilleschen Eigenwertaufgabe, Acta Math. 781946 1-96 MR 7:382d]

[3] G. Borg, Uniqueness theorems in the spectral theory of $y^{\prime \prime}+(\lambda-q(x)) y=0$, Proc. 11th Scandinavian Congr. Math. (Trondheim, 1949), Johan Grundt Tanums Forlag, Oslo, 1952, 276-287. MR 15:315a

[4] E.A Coddington and N. Levinson, Theory of Ordinary Differential Equations, Krieger, Malabar, 1985. MR 16:1022b (earlier ed.) 
[5] A.A. Danielyan and B.M. Levitan, On the asymptotic behavior of the Weyl-Titchmarsh mfunction, Math. USSR Izv. 361991 487-496 MR 92d:34056

[6] R. del Rio, F. Gesztesy, and B. Simon, Inverse spectral analysis with partial information on the potential, III. Updating boundary conditions, Internat. Math. Res. Notices 1997, no.15, 751-758 MR 99a:34032

[7] R. del Rio, F. Gesztesy, and B. Simon, Corrections and addendum to inverse spectral analysis with partial information on the potential, III. Updating boundary conditions, Internat. Math. Res. Notices 1999, no.11, 623-625. CMP 99:14

[8] W.N. Everitt, On a property of the m-coefficient of a second-order linear differential equation, J. London Math. Soc. 41972 443-457 MR 45:7156

[9] F. Gesztesy, H. Holden, B. Simon, and Z. Zhao, Higher order trace relations for Schrödinger operators Revs. Math. Phys. 71995 893-922 MR 97d:34094

[10] F. Gesztesy and B. Simon, Uniqueness theorems in inverse spectral theory for one-dimensional Schrödinger operators, Trans. Amer. Math. Soc. $348 \quad 1996$ 349-373 MR 96e:34030

[11] F. Gesztesy and B. Simon, Inverse spectral analysis with partial information on the potential, I. The case of an a.c. component in the spectrum, Helv. Phys. Acta 701997 66-71 MR 98m:34171

[12] F. Gesztesy and B. Simon, m-functions and inverse spectral analysis for finite and semiinfinite Jacobi matrices, J. d'Analyse Math. 73 (1997), 267-297. MR 99c:47039

[13] O.H. Hald, Inverse eigenvalue problem for the mantle, Geophys. J. R. Astr. Soc. 621980 $41-48$

[14] H. Hochstadt, The inverse Sturm-Liouville problem Commun. Pure Appl. Math. 261973 715-729 MR 48:8944

[15] H. Hochstadt, On the construction of a Jacobi matrix from mixed given data, Lin. Algebra Appl. 281979 113-115 MR 81a:15024

[16] H. Hochstadt and B. Lieberman, An inverse Sturm-Liouville problem with mixed given data, SIAM J. Appl. Math. 341978 676-680 MR 57:10077

[17] K. Iwasaki, Inverse problem for Sturm-Liouville and Hill equations, Ann. Mat. Pura Appl. Ser. 41491987 185-206 MR 89d:34053

[18] S. Jayawardena, The reconstruction of symmetric, anti-symmetric and partially known potentials from spectral data, preprint.

[19] A.Ben Khaled, Problème inverse de Sturm-Liouville associé à un opérateur différentiel singulier, C. R. Acad. Sc. Paris Sér. I Math. 2991984 221-224 MR 86f:34045

[20] M.G. Krein, On the solution of the inverse Sturm-Liouville problem, Dokl. Akad. Nauk SSSR 76 (1951), 21-24 (Russian). MR 12:613e

[21] B. Ja. Levin, Distribution of Zeros of Entire Functions (rev. ed.), Amer. Math. Soc., Providence, RI, 1980. MR 81k:30011

[22] N. Levinson, The inverse Sturm-Liouville problem Mat. Tidskr. B 1949 25-30 MR 11:248e

[23] B. Levitan, On the determination of a Sturm-Liouville equation by two spectra, Amer. Math. Soc. Transl. 68 1968 1-20 MR 28:3194

[24] B. Levitan, Inverse Sturm-Liouville Problems, VNU Science Press, Utrecht, 1987. MR 89b:34001

[25] B.M. Levitan and M.G. Gasymov, Determination of a differential equation by two of its spectra, Russ. Math. Surv. 19:2 (1964), 1-63. MR 29:299

[26] B.M. Levitan and I.S. Sargsjan, Sturm-Liouville and Dirac Operators, Kluwer, Dordrecht, 1991. MR 92i:34119

[27] M.M. Malamud, Similarity of Volterra operators and related questions of the theory of differential equations of fractional order, Trans. Moscow Math. Soc. 551994 57-122 MR 98e:47051

[28] V.A. Marchenko, Some questions in the theory of one-dimensional linear differential operators of the second order, I, Trudy Moskov. Mat. Obšč. 1 (1952), 327-420 (Russian); English transl. in Amer. Math. Soc. Transl. (2) 101 (1973), 1-104. MR 15:315b

[29] V. Marchenko, Sturm-Liouville Operators and Applications, Birkhäuser, Basel, 1986. MR 88f:34034

[30] A.I. Markushevich, Theory of Functions of a Complex Variable (2nd ed.), Chelsea, New York, 1985. MR 56:3258 (earlier ed.)

[31] J. Pöschel and E. Trubowitz, Inverse Scattering Theory, Academic Press, Boston, 1987. MR 89b:34061 
[32] M. Reed and B. Simon, Methods of Modern Mathematical Physics IV, Analysis of Operators, Academic Press, New York, 1978. MR 58:12429c

[33] R. del Rio Castillo, On boundary conditions of an inverse Sturm-Liouville problem, SIAM J. Appl. Math. 501990 1745-1751 MR 91j:34023

[34] W. Rundell and P.E. Sacks, Reconstruction techniques for classical inverse Sturm-Liouville problems, Math. Comp. 581992 161-183 MR 92h:34034

[35] T. Suzuki, Deformation formulas and their applications to spectral and evolutional inverse problems, Nonlinear Partial Differential Equations in Applied Science (Tokyo, 1982), NorthHolland Math. Stud., vol. 81, North-Holland, Amsterdam, 1983, pp. 289-311. MR 85j:35196

[36] T. Suzuki, Gel'fand-Levitan's theory, deformation formulas and inverse problemsJ. Fac. Sci. Univ. Tokyo Sect. IA, Math. 321985 223-271 MR 86k:35157

[37] T. Suzuki, Inverse problems for heat equations on compact intervals and on circles, I, J. Math. Soc. Japan 381986 39-65 MR 87f:35241

[38] E.C. Titchmarsh, Eigenfunction Expansions, Part I (2nd ed.), Clarendon Press, Oxford, 1962. MR 31:426

Department of Mathematics, University of Missouri, Columbia, Missouri 65211

E-mail address: fritz@math.missouri.edu

Division of Physics, Mathematics, and Astronomy, California Institute of Technology, Pasadena, California 91125

E-mail address: bsimon@caltech.edu 